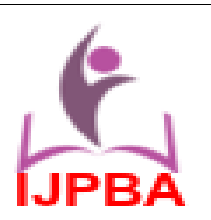

Contents lists available at www.ijpba.in

International Journal of Pharmaceutical and Biological Science Archive

NLM (National Library of Medicine ID: 101738825 )

Index Copernicus Value 2019: 71.05

Volume 9 Issue 3; May-June; 2021; Page No. 13-22

\title{
IONTOPHORESIS: A FUNCTIONAL APPROACH FOR ENHANCEMENT OF TRANSDERMAL DRUG DELIVERY
}

\author{
${ }^{1}$ Sanjoy De, ${ }^{2}$ Souvik Mallik, ${ }^{3}$ Sabuj Kumar Bhattacharya, ${ }^{4}$ Shibam Acharya, ${ }^{5}$ Partha Sarathi \\ Mondal, ${ }^{6}$ Soumya Rakshit, ${ }^{7}$ Bankim Chandra Nandy \\ ${ }^{1-6}$ Students of School of Pharmacy, Techno India University, Kolkata, West Bengal, India. \\ ${ }^{7}$ Associate Professor, School of Pharmacy, Techno India University, Kolkata, West Bengal, India.
}

Conflicts of Interest: Nil

Corresponding author: Sanjoy De

DOI: https://doi.org/10.32553/ijpba.v9i3.190

\begin{abstract}
Iontophoresis is one of the most widely studied active techniques for enhancing transdermal delivery of drugs. However, its ability to enhance the delivery of highly lipophilic compounds is poor due to lack of any charge and poor water solubility of molecules. The skin has been used as a port for systemic delivery of therapeutic agents since several decades. The composition of stratum corneum renders it a daunting barrier to the topical and transdermal administration of therapeutic agents. The number of drug molecules for transdermal delivery is limited owing to the physicochemical restrictions.

The delivery of drugs into systemic circulation via skin has generated much attention during the last decade. Transdermal therapeutic systems propound controlled release of active ingredients through the skin and into the systemic circulation in a predictive manner. Drugs administered through these systems escape first-pass metabolism and maintain a steady state scenario similar to a continuous intravenous infusion for up to several days.
\end{abstract}

Keywords: Iontophoresis, non-invasive, stratum corneum, acid-alkaline reaction, chemical permeation enhancer, reverse iontophoresis.

\section{Introduction}

The skin is the largest organ of the human body, with surface area of about $2 \mathrm{~m}^{2}$. Historically, the skin was viewed as an impermeable barrier but in recent years, it has been increasingly recognized that intact skin can be used as a port for topical or continuous systemic administration of drugs [1].

And Skin is one of the most extensive and readily accessible organs of the human body. In modern-day pharmaceutical practice, therapeutic compounds are applied to the skin for dermatological (within the skin), local (regional) and transdermal (systemic) delivery. For transdermal delivery of drugs, stratum corneum is the main barrier layer for permeation of drug [2].

The benefits of using transdermal drug delivery include improved systemic bioavailability resulting from bypassing the first metabolism. Variables due to oral administration, such as $\mathrm{pH}$, the presence of food or enzymes and transit times can all be eliminated. In the development of new transdermal drug delivery devices the aim is to obtain controlled, predictable and reproducible release of drugs into the blood stream of the patient [3].

Iontophoresis provides the usual advantages of a trandermal route like, therapeutic efficacy improvement by bypassing hepatic "first pass" metabolism, avoidance of inconvenience caused by parenteral drug delivery and prevention of variation in the absorption seen with oral administration. Besides this, it also reduces the chance of dosing variation by providing pro-grammed delivery of the drug. Iontophoresis also provides a therapeutic 
regimen leading to better patient compliance. It permits the use of a drug with a short biological half life since the drug is delivered to the target area without the need to recirculate in the blood. Moreover, the drug is delivered into the bloodstream directly without any delay $[4,5]$.

This technique not only provides usual benefits of transdermal delivery but it can also be used for programmed and controlled delivery of drugs by adjusting the current, as the flux of drug into the system is in proportion to the current. This not only avoids dependence on biological variables but also improves patient compliance. In iontophoresis, a small electric current forces molecules into the skin. An electrode patch containing the drug is placed on the skin and this acts as the working electrode.

Historical background of iontophoretic process: Iontophoresis, derived from the Greek "ionto" meaning 'ion' and "phoresis" meaning 'to bear,' is a process that allows increased penetration of ionized molecule across or into the tissue by application of low electric current. Clinical application of current can be traced back to the ancient time of the golden age of the Greek civilization and was probably originated by Varatti in 1747 [6].

The two scientists in the 18 th century used the knowledge of electricity that it can move different metal ions, and that the movement of the ions produce electricity. According to Helmstadter, increasing progress was made in the 19 th century notably by William James Morton (1846-1920), Stephen Leduc (1853-1939) and Fritz Frankenhauser (1868) on electromotive administration of metal ions and alkaloids. At 20 th century, the method of administering pharmacological agents by iontophoresis became popular due to the work of Leduc (1900) who introduced the term 'iontotherapy' and formulated the laws for this process [7].

This technique not only provides usual benefits of transdermal delivery but it can also be used for programmed and controlled delivery of drugs by adjusting the current, as the flux of drug into the system is in proportion to the current. This not only avoids dependence on biological variables but also improves patient compliance [8-13]. In iontophoresis, a small electric current forces molecules into the skin. An electrode patch containing the drug is placed on the skin and this acts as the working electrode.

This technique not only provides usual benefits of transdermal delivery but it can also be used for programmed and controlled delivery of drugs by adjusting the current, as the flux of drug into the system is in proportion to the current. This not only avoids dependence on biological variables but also improves patient compliance This technique not only provides usual benefits of transdermal delivery but it can also be used for programmed and controlled delivery of drugs by adjusting the current, as the flux of drug into the system is in proportion to the current. This not only avoids dependence on biological variables but also improves patient compliance [9-13]. In iontophoresis, a small electric current forces molecules into the skin. An electrode patch containing the drug is placed on the skin and this acts as the working electrode. This can be either positive or negative, depending on the characteristics of the drug.

\section{AN OVERVIEW OF IONTOPHORESIS:}

Iontophoresis is the method where the movements of ions across a membrane enhanced using an externally applied potential difference. When the membrane under consideration is skin, the method is called transdermal iontophoresis [2].

The highly lipophilic nature of the skin restricts the permeation of hydrophilic, high molecular weight and charged compounds through the stratum corneum into the systemic circulation. However, many therapeutically active drug molecules are hydrophilic and possess high molecular weights for example, peptides [5].

\section{PRIINCIPAL OF IONTOPHORESIS:}

It is based on the mechanism of current flow, like charges repels each other while unlike get attracted. The positive charged ions in the solution are repelled from positive charged electrode which is positioned on the tissue on which the drug molecules has to be delivered, similar way for the negative ions at negative electrode. The direct current is used for the transfer of the ions from the drug electrode. The external energy is used to increase the rate of drug delivery. Neutral molecule moves by connective flow due to electro osmotic and osmotic force by the application of current $[8,9]$.

The term IONTOPHORESIS simply defined as ion transfer $($ ionto $=$ ion $\&$ phoresis $=$ transfer) $[10]$

Electro migration of ions during iontophoresis causes convective solvent motion and this solvent motion in turn 'drags' neutral or even charged molecules along with it. This process is termed as electro-osmosis. At pH values above 4, the skin is negatively charged, implying that positively charged moieties like $\mathrm{NaCl}$ molecules will be more easily 
transported as they attempt to neutralize the charge in the skin to maintain electro neutrality [11].

The drug formulation(D+ A-) containing the ionized molecule (D+) which is placed in the electrode + (anode) compartment bearing the same charge. The electrode compartment is placed at a distal site on the skin; the following figure shows Iontophoresis device using a $\mathrm{Ag} / \mathrm{AgCl}$ electrode system.

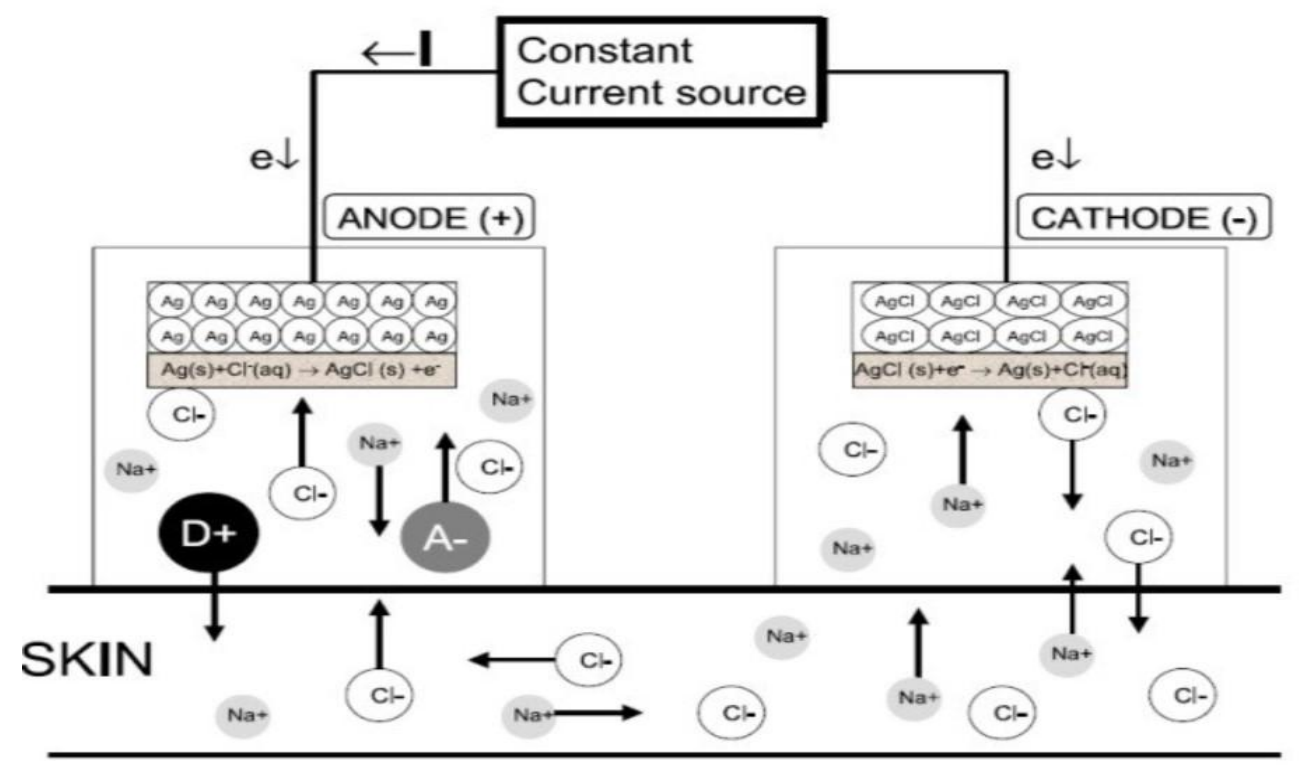

Fig.1: Iontophoresis device using an $\mathrm{Ag} / \mathrm{AgCl}$ electrode system [12]

The $\mathrm{Cl}$ - ions arrive at the electrode solution interface and react with the metallic silver to form $\mathrm{AgCl}$. Because of $\mathrm{Ag} \mathrm{Cl}$ - low solubility product, it will be deposited at the electrode surface, simultaneously releasing an electron.

- In order to maintain electroneutrality in the anodal compartment, either a cation must move out of the compartment and into the skin or an anion must leave the skin and move into the anodal chamber.

- In the cathodal compartment, the $\mathrm{AgCl}$ is reduced by the arrival of electrons from the power supply and yields metallic silver together with a Clion, which passes into the solution.
- Again, for electro neutrality, this must be compensated for by the arrival of a cation from within the skin into the cathodal chamber or by the loss of an anion.

Therefore the $\mathrm{Na}+$ and $\mathrm{Cl}$ - inside the skin can impact on the efficiency of drug transport when the electrical circuit is completed.

In result the Iontophoresis drug delivery method is using external electrical potential difference to facilitate movement of drug ions across the skin [12].

\section{The positively charged chamber, called the anode, will repel a positively charged chemical into the skin.}

\section{The negatively charged chamber, called the cathode, will repel a negatively charged chemical into the skin.}




\section{Acid/Alkaline Reactions:}

Will get ACID accumulation under the POSITIVE (anode) electrode (weak hydrochloric acid) because the negatively charged chloride ions $(\mathrm{Cl}$-from $\mathrm{NaCl})$ will transit (be attracted) towards the anode. Will get ALKALINE accumulation under the NEGATIVE (cathode) electrode (SODIUM HYDROXIDE) because the positively charged sodium ions $(\mathrm{Na}+$ from $\mathrm{NaCl}$ )will move towards the cathode. The $\mathrm{Na}+$ ions react with water to form sodium hydroxide $(\mathrm{NaOH})$. N.B. it is suggested that the reaction at the negative (cathode) electrode will bring about a softening of the skin, hence is growing use by beauty therapy clinics [12].

The drug is applied under an electrode of the same charge as the drug, and a return electrode opposite incharge to the drug is placed at a neutral site on the body surface [8].

\section{Merits: [13-15]}

- The benefit of iontophoretic drug delivery in ophthalmology lay in its capacity to obtain high intraocular and especially posterior pole drug tissue concentration in a controlled and safe fashion, while minimizing the systemic drug exposure.

- The application of iontophoresis as a noninvasive and local drug administration method has the advantage of a drug delivery.

- It bypasses the hepatic first pass effect and gastrointestinal vagaries.

- Iontophoresis facilitates the transport of charged molecules and even of those with higher molecular weight.

- Re-usable ion-chambers: drugs, adhesive discs and batteries are the only consumables.

- Easy termination of drug delivery in case of toxicity.

- Offering better control over the quantity of drug delivered since the amount of drug delivered depends on applied current, duration of applied current, and area of skin exposed to the current.

- Prevent variation in the absorption and metabolism as seen with oral administration.

- Simple non invasive administration and improved bioavailability.

- Enhancing the delivery of polar molecules as well as high molecular weight compounds.

- Enhanced safety, efficacy, reliability and acceptability.

\section{Demerits:[13-15]}

- $\quad$ Side effects such as erythema, skin irritation or minor burns were observed in iontophoretic applications in dermatology.

- During iontophoretic transport across the skin, the drug encounters potential of hydrogen $(\mathrm{pH})$ differences between 4 to 7.3 , leading to the possibility of the drug molecule becoming uncharged and losing the major effect of electric field.

- Iontophoresis cannot achieve high drug level in blood.

- Diseased skin as well as extent of disease can affect penetration.

- Iontophoresis is not suggested for under arm or facial / head hyperhydrosis.

- The metabolic enzyme in the skin can increase the problem.

- Iontophoresis is restricted to drugs that can be formulated in ionized form.

- Skin pigmentation can occur with longer application of some drugs (e.g. sodium nitroprusside can stain brown) butnormally subsides after a few weeks.

- Minor skin damage (including burns) can occur when higher currents are used. This has been observed in andaround sweat ducts.

\section{REVERSE IONTOPHORESIS:}

Reverse iontophoresis, a technique in which low electric current is applied to draw intestinal fluid through the skin, is widely applied now a days in devices meant for diagnostic application. This provides a convenient and non-invasive method for sampling of body fluids so as to permit simultaneous measurement of the desired substance in the body fluid and thus to monitor them efficiently e.g., devices like Glucowatch ${ }^{\circledR}$ uses the reverse iontophoretic process to continuously monitor the glucose level in the blood. This system provides a needleless means of monitoring blood glucose levels in diabetic patients and uses an electrical signal that is proportional to the amount of glucose in the extracellular fluid [16].

Glucose can be extracted through intact skin by electro-osmotic flow (a process called 'reverse iontophoresis') upon the application of a low-level electrical current. Recently we have combined iontophoretic extraction with an in situ glucose sensor in a device called the GlucoWatch biographer. Clinical results with this device showed close tracking of blood glucose over a range of 2.2 to $22.2 \mathrm{mmol} / \mathrm{l}$ for up to $12 \mathrm{~h}$ using a single blood glucose value as calibration. The biographer 
readings lag behind blood glucose values by an average of $18 \mathrm{~min}$. An analysis of data from 92 diabetic subjects in a controlled clinical setting shows a linear relationship $(\mathrm{r}=0.88)$ between GlucoWatch biographer readings and blood glucose. The mean absolute relative difference between the two measurements was $15.6 \%$ and more than $96 \%$ of the data fell in the $(\mathrm{A}+\mathrm{B})$ regions of the Clarke error grid. Similar results have been obtained from subjects using the GlucoWatch biographer in an uncontrolled home environment. The automatic, frequent, and non-invasive measurements obtained with the GlucoWatch biographer provide substantially more information about glucose levels than do the current finger stick methods. This information can be used for improved decisions about all aspects of diabetes management [20].The GlucoWatch technology requiring calibration with traditional finger-stick glucose measurements is able to provide readings every $20 \min$ for $12 \mathrm{~h}$. This is a patient friendly mechanism as regular finger pricks are avoided. GlucoWatch is approved for use in children and adults, and is currently indicated only as an adjunctive therapy to conventional blood glucose monitoring. The technique of reverse iontophoresis provides a feasible method for rapid, linear extraction of phenylalanine and for easy detection (by instrument like biosensors) of monitoring diseases like phenylketonuria [16].

\section{Criteria for Iontophoresis:[17,18]}

There are some very important rules that have to be followed when doing Iontophoresis:

1. The selected molecule must be ionized into positive and negative components and be maintained as ions during the treatment.

2. The size of the ion is important. For example even though a complex protein like collagen may be possible to ionize, due to large size of the important ion of collagen it cannot be transported through skin.

3. There is a limit to the number of polar substances that can be used simultaneously. If there are too many charged particles then the pores may be blocked by the crowd of ions converging all at once [17].

4. As electricity is only conducted through water and not through lipids, the ion must be watersoluble.
5. The $\mathrm{pH}$ of the active gel is of fundamental importance because $\mathrm{pH}$ is responsible for the ionization therefore $\mathrm{pH}$ at which $100 \%$ ionization take place considered as a ideal $\mathrm{pH}$.

6. The current used should be effective one. It is high enough to be effective that it will move the ions effectively but still safe.

7. Pulsatile current works better than continuous current because it will increase the magnitude of effective electric current by allowing skin to convert from polarized state to depolarized one periodically [18].

\section{Chemical Enhancers in Iontophoresis:}

For a transdermal delivery system to be successful, it should make the drug permeate through the skin to the sys-temic circulation in quantities sufficient to show the thera-peutic effect. In many cases, iontophoresis itself has been able to show an increased permeation of the drug molecule but, in many others, iontophoresis alone has not given desired results which have lead to the use of various chemical enhancers along with iontophoresis to enhance the delivery of drugs especially larger peptide molecules [4]. An ideal enhancer must be nontoxic, nonirritating, nonallergenic, pharmaceutically inert and compatible with most drugs and excipients. Azone and oxazolidinedione are considered to be among the most promising enhancers. However, no single agent meets all the desirable attributes of an enhancer. A combination of enhancers may thus be required. For most chemical enhancers, the strength of activity depends on their concentration. Toxicity of enhancers may limit their use in transdermal formulation. There are evidences of showing synergistic effects between the chemical enhancers and iontophoresis. e.g. buspirone hydrochloride [19], atenolol [20], nicorandil [21], nicardipine [22]. Fatty acids and terpenes are probably the most studied chemical enhancers for promoting iontophoretic drug delivery. However, synergistic effects of iontophoresis of some other enhancers have also been reported. Dimethyl acetamide (DMA) was found to be able to severely compromise skin barrier properties and enhance iontophoretic delivery of insulin [23-34]. The effects of some solvents used [EtOH, ethyl acetate (EtAC), isopropyl myristate (IPM) and PG] in insulin iontophoresis were also investigated. All the solvents produced synergistic enhancement with iontophoresis [35]. 


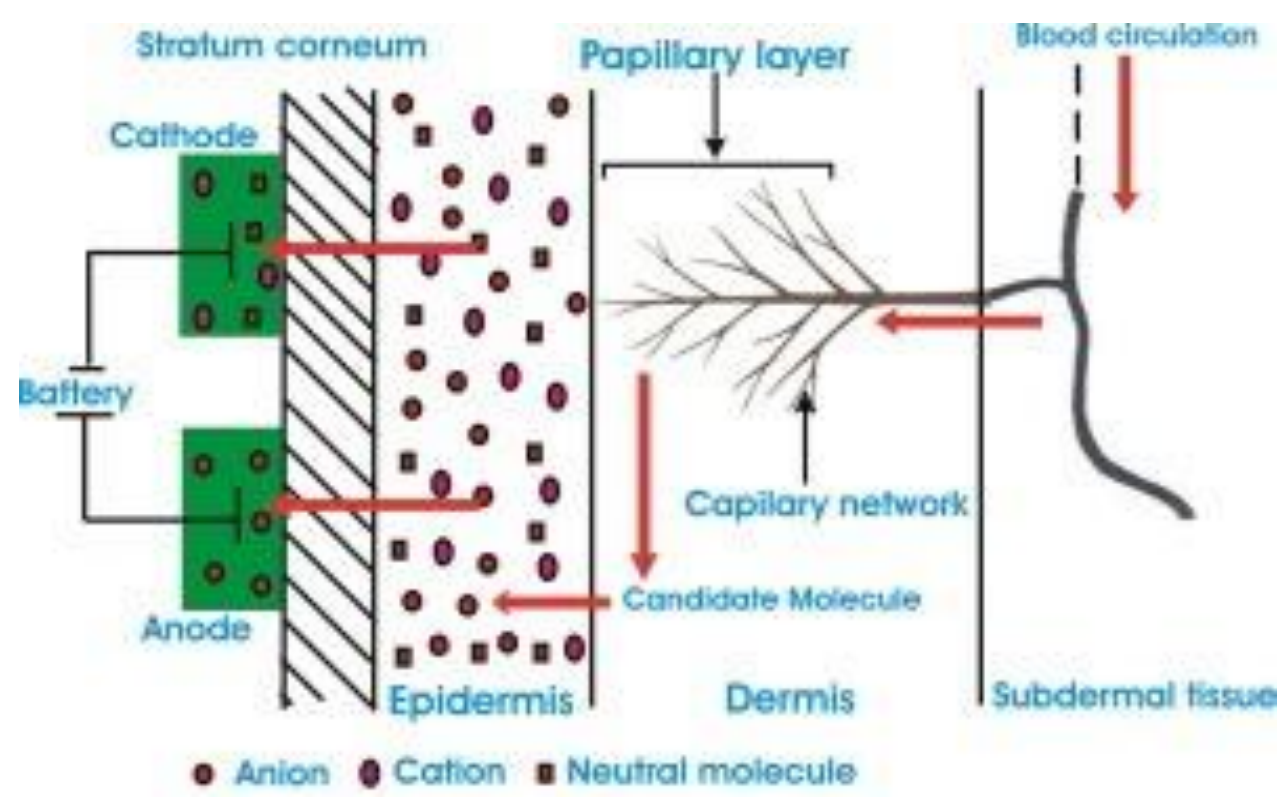

Fig 2: Reverse Iontophoretic extraction of candidate molecule [23]

\section{Factors Affecting Iontophoresis Transport System:}

Human skin is not all the same. There are numerous differences among patient groups as well as between various regions of the body, age and ethnicity. Various factors have been shown to affect the results of iontophoresis. The following factors have to be considered [25,26].

\section{pH of the Drug Solution:}

Drug solution $\mathrm{pH}$ will have a very significant influence on iontophoretic delivery of drug from the formulation. Changes in the $\mathrm{pH}$ of the fluid at the driving electrode can influence the transport. The $\mathrm{pH}$ can determine whether or not the drug is charged or it can affect the ratio of the charged and uncharged species. For the delivery of polypeptides, the type of charge is also controlled by the formulation $\mathrm{pH}$ relative to the isoelectric point of the polypeptide [27].

When the $\mathrm{pH}$ decreased, the concentration of hydrogen ion increases and a vascular reaction (vasodilatation) is initiated because of $\mathrm{C}$-fiber activation, thus it is important to keep the $\mathrm{pH}$ as close as possible to and, at least when working with vasodilators, at $\mathrm{pH} 5.5$ and below. There is an increasing risk for vascular reaction due to the high concentration of hydrogen ions rather than the compound used. Since hydronium ions are small they penetrate the skin more easily than larger drug ions. Laboratory findings vary on the effect of $\mathrm{pH}$ and drug behavior. According to the HendersonHasselbalch equation, $\mathrm{pH}$ is the determining factor governing the amount of drug present in the ionized state [28].

\section{Drug concentration:}

The type of the drug molecule used, the steady state flux (drug ion movement) has been reported to increase with increasing concentration of the solute drug molecule in the donor chamber. i.e. In the drug delivery electrode. A limiting range to be considered is the strength of the current applied. At the high level drug ion concentrations, the movement may become independent of concentration, due to reason of the saturation of the skin boundary layer relative to the donor bulk solution [29].

\section{Molecular size:}

It has been shown that the permeability coefficients in positively charged, negatively charged and uncharged solutes across human skin are a function of molecular size. When the molecular size increases, the permeability coefficient decreases. However, there are certain solutes with a relatively high molecular size (e.g. insulin, vasopressin and several growth hormones), which have also been to penetrate the skin barrier into the systemic circulation [30].

\section{Current strength:}

There is a linear relation between the observed fluxes of a $1-\mathrm{cm}^{2}$, the current is limited to $1 \mathrm{~mA}$ due to patient comfort considerations. This current should not be applied for more than 3 min because of local skin irritation and burns. With increasing current, the risk of non specific vascular reactions (vasodilatation) increases. At a current of 0.4-0.5 
$\mathrm{mA} / \mathrm{cm}^{2}$, such a vascular reaction is initiated after a few seconds of iontophoresis with deionised or tap water. This latter effect is probably due to current density being high enough a small area to stimulate the sensory nerve endings, causing reactions such as the release of substance $\mathrm{P}$ from $\mathrm{C}$-fiber terminals $[31,32]$

\section{Ionic strength:}

The ionic strength of drug delivery system is proportional to the Iontophoretic permeation of drugs. Some authors reported that increasing the ionic strength leads to decreases the rate of permeation and has no significant effect on penetration up to the $0.5 \mathrm{~cm}^{2}$.

\section{APPLICATIONS OF IONTOPHORESIS:}

Iontophoresis has been used for the treatment of various dermatologic conditions [Table-1] The majority of published studies are either uncontrolled series or anecdotal observations. Earlier, simple ions and heavy metals were the most frequently used drugs, but over the last 30 years interest has shifted toward the use of iontophoresis as a drug delivery system for a wide variety of medications, ranging from steroids to antibiotics to local anesthetics .

Table 1: Summary of conditions treated via iontophoresis [35-40]

\begin{tabular}{|l|l|}
\hline Condition & Drug used \\
\hline Ischemic ulcer & Zinc oxide \\
\hline Ulcers & Histamine \\
\hline Plantar warts & Sodium salicylate \\
\hline Herpes simple & Idoxuridine \\
\hline Aphthous stomatitis & Triamcinolone acetonide \\
\hline Lichen planus & Methyl prednisone \\
\hline Hyperhidrosis & Poldine methyl sulfate, glycopyrroniumbromide, atropine, tap water \\
\hline
\end{tabular}

\section{Hyperhidrosis:}

The most successful application of iontophoresis is for the treatment of hyperhidrosis [36]. Hyperhidrosis (hi-pur-hi-DROE-sis) is abnormally excessive sweating that's not necessarily related to heat or exercise. You may sweat so much that it soaks through your clothes or drips off your hands. Besides disrupting normal daily activities, this type of heavy sweating can cause social anxiety and embarrassment [37]. The most successful application of iontophoresis is for the treatment of hyperhidrosis. The basis for such treatment and its practical aspects have been well described [38],[39].
Tap water iontophoresis is one of the most popular treatments used in this condition because it is safe and effective. The procedure uses a mild electrical current that is passed through tap water to temporarily shut off sweat glands. According to one hypothesis, iontophoresis may induce hyperkeratosis of the sweat pores and obstruct sweat flow and secretion (although no plugging of the pores has been found. Successful induction of hypohydrosis by tap-water iontophoresis requires the application of $15-20 \mathrm{~mA}$ to each palm or sole for $30 \mathrm{~min}$ per session for 10 consecutive days, followed by one or two maintenance sessions per week [40].

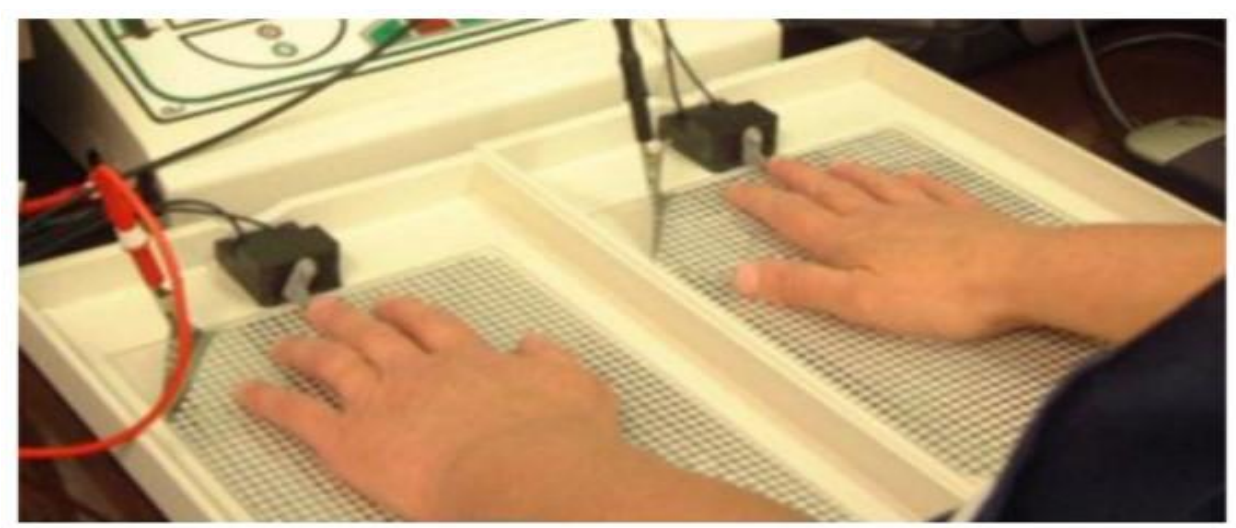

Fig 3: Treatment on Hyperhydrosis [33] 


\section{Ophthalmology:}

Iontophoresis has been used experimentally to deliver antibiotics into the eye. The principal disadvantage of this technique is the time required for direct contact of the electrode with the eye. Iontophoresis of various drugs like atropine,scopolamine, sulfadiazine, gentamycin, fluorescein etc [41].

\section{Otorhinolaryngology:}

Iontophoresis is a preferred method for obtaining anesthesia of the tympanic membrane prior to simple surgical procedures involving that structure. Iontophoresis of zinc has also been used for the treatment of patients with allergic rhinitis [42]. Anesthesia of the skin can be achieved with the use of a variety of positive and negative controls, including iontophoresis of epinephrine and lidocaine separately, and topical administration of lidocaine and epinephrine [43].

\section{Conclusion:}

It should be evident from this review article that iontophoresis hold a lot of commitment for the future of drug delivery. Iontophoresis has been explored for many dermatologic (e.g: Transdermal Drug Delivery System) and other medical conditions with reports of considerable success. Iontophoresis, this technique with other enhancement of transdermal drug delivery system have been widely investigated in recent years. Without iontophoresis, such charged species are not able to penetrate the skin due to lipophilic nature of the skin and it easily penetrates in the corneum barrier of the skin. These techniques are non-invasive and convenient for drug delivery system due to its good bioavailability profile, short half life and multiple dosing schedules. Rate of drug input can be controlled and optimized easily in this drug delivery system. Thus, iontophoresis may become an important alternative method of drug delivery in the near future.

\section{Reference:}

1. Prabhakar Panzade, Prashant Puranik. Iontophoresis: A Functional Approach for Enhancement of Transdermal Drug Delivery. Asian Journal of Biomedical \& Pharmaceutical Science.2012;11(2):1-2.

2. Swati rawat, Sudha vengurlekar, B. Rakesh, S. Jain, G. Srikarti. Transdermal Drug Delivery Of Iontophoresis. Indian J Pharm Sci. 2008 Jan-Feb; 70(1): 5-10.

3. Vinod Dhote, Punit Bhatnagar, Pradyumna K. Mishra, Suresh C. Mahajan, and Dinesh K. Mishra . Iontophoresis: A Potential
Emergence of a Transdermal Drug Delivery System. Sci Pharm. 2012 Jan-Mar; 80(1): 128.

4. Nitin Dixit, Vikas Bali, Sanjula Baboota, Alka Ahuja and Javed Ali. Iontophoresis - An Approach for Controlled Drug Delivery: A Review. Bentham Science Publishers Ltd. 2007; 4(1): 1-10.

5. Azad Khan, Mohd Yasir, Mohd Asif, Iti Chauhan, Alok P. Singh, Rajat Sharma, Pradeep Singh and Shubham Rai. Iontophoretic drug delivery: History and applications. Journal of Applied Pharmaceutical Science. 2011; 01 (03):11-24.

6. Anil Kumarj. Shinde, Amit. Shinde, Kevinc. Garala, Sachina. Kandekar, Harington. Physical penetration enhancement by iontophoresis : a review. Int. Journal of Curr. Pharmaceutical Research, Vol 2, Issue 1, 2010, Pharmazie.2001;56(7):583-7.

7. Smirithi Sharma, Nayyar Parvez and Pramod Kumar Sharma. Iontophoresis-Models and Applications: A Review. African Journal of Basic \& Applied Sciences. 2015; 7(1):1-7.

8. https://www.linkedin.com/pulse/iontophoresis -drug-delivery-dr-eng-walid-tarawneh

9. R.R. Burnette, B. Ongpipattanakul, Characterization of the permse-lective properties of excised human skin during iontophoresis, J. Pharm. Sci. 76 (1987) 765773

10. Hardainiyan, Swati \& Bankim, Chandra \& Nandy, \& Jasuja, Dr. Nakuleshwar \& Vyas, Pritesh \& Raghav, Pramod. A Review on The Recent Innovations in Transdermal Drug Delivery for Herbal Therapy. Journal of Biomedical and Pharmaceutical Research. 3, 2014, 88-101.

11. https://www.linkedin.com/pulse/iontophoresis -drug-delivery-dr-eng-walid-tarawneh

12. Bankim Chandra Nandy, Bhaskar Mazumder et al. Transdermal Electronically Assisted Technologies: Current Approaches on Iontophoretic Delivery System. Int Journal of Drug Formul and Res.2011: 2 (4), 1-31.

13. Iontophoresis with Laser Doppler Assessment of Blood FlowResponse, Issue 1 https://www.moor.co.uk/wpcontent/uploads/2018/10/Moor_Iontophoresis _Theory_Issue_1.pdf

14. Merino, V.; López, A.; Hochstrasser, D.; Guy, R.H. J. Control. Release 1999, 61(1-2), 65-9.

15. Applications of Iontophoresis and Phonophoresis by Craig Hahn Bachelor of 
Science in Physical Therapy University of North Dakota, 1995 https://core.ac.uk/download/pdf/235074193.p df

16. Iontophoresis: controlled transdermal drug delivery system N. Kirubakaran, M. Chandrika and K. Reeta Vijaya Rani Himalaya Drug Company, Makali, Bangalore, Karnataka, IndiaPeriyar College of Pharmaceutical Sciences, Trichy, Tamil Nadu, India https://ijpsr.com/bft-article/ iontophoresis-controlled-transdermal-drugdelivery-system/?view=fulltext

17. Swapnil Deshpande, Swaroop Lahoti, Rohit Shah, Madhuri Shinde, Sagar Motarwar, Chaitrali Pawar. Iontophoresis: A Physical Approach to Transdermal Drug Delivery System Research J. Pharm. and Tech. 5(2): Feb. 2012; Page 175-180.

18. Meiden VM, Mohammad A, Michniak BB. Enhanced iontophoretic delivery of buspirone hydrochloride across human skin using chemical enhancers. Int J Pharm. 2003; 264: 73-83.

19. Bhaskaran S, Harsha SN. Effect of permeation enhancer and iontophoresis on permeation of atenolol from transdermal gels. Indian J Pharm Sci. 2000; 6: 424-426

20. Nandy B. C., Gupta R. N., et al. Transdermal Iontophoretic Delivery of Atenolol in Combination with Penetration Enhancers: Optimization and Evaluation on Solution and Gels, International Journal of Pharmaceutical Sciences and Drug Research 2009; 1(2): 9199

21. Krishnaiah YSR, Satyanarayana V, Bhaskar P. Enhanced Percutaneous Permeability of Nicardipine Hydrochloride by Carvone Across the Rat Abdominal Skin. Drug Dev Ind Pharm. 2003; 29 (2): 191-202.

22. Tapan Kumar Giri, Subhasis Chakrabarty,Bijaya Ghosh, Transdermal reverse iontophoresis: A novel technique for therapeutic drug monitoring, https://www.sciencedirect.com/science/article /abs/pii/S0168365916308550\#!

23. https://www.sciencedirect.com/science/article /abs/pii/S0168365916308550

24. Russell O Potts, Janet A Tamada, Michael J Tierney.Glucose monitoring by reverse iontophoresis.2002; 18(1):49-53.
25. Chien YW. Development of Transdermal drug delivery systems. Drug Dev Ind Pharm. 1987; 13:589-651.

26. Srinivasan V, Higuchi WI. A model for iontophoresis incorporating the effect of convective solvent flow. Int J Pharm. 1990; 60:133-138.

27. Iontophoretic Drug Delivery by Ramesh Gannu1 and Y. Madhusudan Rao. http://bspublications.net/downloads/053d8e54 424d76_advances\%20in\%20drug\%20delivery -chapter-1.pdf

28. Fang JY, Hung CF, Wong WW. Skin Pharmacol Physiol. 2006;19:28-37.

29. N. Kirubakaran, M. Chandrika and K. Reeta Vijaya Rani. Iontophoresis: controlled transdermal drug delivery system https://ijpsr.com/bft-article/iontophoresiscontrolled-transdermal-drug-deliverysystem/?view=fulltext

30. Chien YW, Siddiqui O, Shi W, Lelawongs P, Liu JC. Direct current iontophoretic Transdermal delivery of peptides and protein drugs. J Pharm Sci. 1989;78:377.

31. Abramowitz D, Neoussikine B. Treatment by Ion Transfer. New York: Grune and Stratton; 1946. p. 87.

32. Schriber WJ, editor. A manual of electrotherapy. 4th ed. Philadelphia: Lea and Febiger; 1975. pp. 125-31.

33. Lohe R. G., Jadkar S. S., Modekar S. D. and Bhusare K. M. Iontophoresis drug delivery system. World Journal of Pharmacy and Phamaceutical Science. 2016; 5 (9):P-753. https://storage.googleapis.com/journaluploads/wjpps/article_issue/1472634883.pdf

34. O. Pillai, V. Nair, R. Panchagnula, Transdermal iontophoresis of insulin: IV. Influence of chemical enhancers, Int. J. Pharm. 269 (2004) 109-120.

35. Yiping Wanga, Rashmi Thakura, Qiuxi Fan, Bozena Michniak, Transdermal iontophoresis: combination strategies to improve transdermal iontophoretic drug delivery. European Journal of Pharmaceutics and Biopharmaceutics 60 (2005) 179-191.

36. Reena Rai, C. R. Srinivas. Iontophoresis in dermatology. Indian J Dermatol Venereol Leprol Jul-Aug 2005; 71(4):236-239.

37. https://www.mayoclinic.org/diseasesconditions/hyperhidrosis/symptoms-causes/ syc-20367152 
38. Grice K. Hyperhidrosis and its treatment by iontophoresis. Physiother 1980; 66:43-4.

39. Morgan K. The technique of treating hyperhidrosis by iontophoresis. Physiother 1980; 66:45.

40. Rathi AA, Dhamecha DL. Physical approaches to penetration enhancement. International journal of pharmatech research, 2010; 2: 2364-2378.

41. V. Srinivasan, W.I. Higuchi, A model for iontophoresis incorporating the effect of convective solvent flow, Int. J. Pharm. 1990; 60: 133-198.
42. Azad Khan, Mohd Yasir, Mohd Asif, Iti Chauhan, Alok P. Singh, Rajat Sharma, Pradeep Singh and Shubham Rai. Iontophoretic drug delivery: History and applications. Journal of Applied Pharmaceutical Science 01 (03); 2011: 11-24.

43. Gangarosa LP Sr. Defining a practical solution for iontophoretic local anesthesia of skin. Methods Find Exp Clin Pharmacol 1981; 3:83-94. 